\title{
On the Initial Value Problem of the Second Painlevé Transcendent
}

\author{
A. S. Fokas and M. J. Ablowitz \\ Department of Mathematics and Computer Science, Clarkson College of Technology, Potsdam, \\ NY 13676, USA
}

\begin{abstract}
The initial value problem associated with the second Painlevé Transcendent is linearized via a matrix, discontinuous, homogeneous Riemann-Hilbert $(\mathrm{RH})$ problem defined on a complicated contour (six rays intersecting at the origin). This problem is mapped through a series of transformations to three different simple Riemann-Hilbert problems, each of which can be solved via a system of two Fredholm integral equations. The connection of these results with the inverse scattering transform in one and two dimensions is also pointed out.
\end{abstract}

\section{Introduction}

At the turn of the century Painlevé [1] and his school [2] classified all equations of the form $q_{t t}=F\left(q_{t}, q, t\right)$, where $F$ is rational in $q_{t}$, algebraic in $q$ and locally analytic in $t$, which have the Painlevé property, i.e. their solutions are free from movable critical points [3]. Distinguished amongst these fifty equations are the so-called six Painlevé transcendents, P I-P VI; any other of the fifty equations can either be integrated in terms of known functions or can be reduced to one of these six equations.

In the Soviet literature [4] interesting results regarding exact solutions and properties of the Painlevé transcendents are summarized in [5]; the main results are : i) For a certain choice of their parameters, P II-P V admit rational solutions, as well as one-parameter families of solutions expressible in terms of elementary transcendental functions (Airy, Bessel, Weber-Hermite, Whittaker, respectively). ii) P II-P V admit transformations [6-9] which map solutions of a given Painlevé equation to solutions of the same equation, but with different values of its parameters. These transformations can be used to generate recursively the solutions mentioned in i). Similar results have also been obtained for P VI [10].

In recent years further interest in the Painlevé equations has developed due to the following reasons: i) Although PI-VI were first discovered from strictly mathematical considerations, they have recently appeared in several physical 
applications (see for example [11-14]). ii) Ablowitz et al. [15] have discovered a remarkable connection between equations with the Painleve property and PDE's solvable by the inverse scattering transform (IST). For example, P II and special cases of P III and P IV can be obtained from the exact similarity reduction of the modified Korteweg-deVries, the sine-Gordon and the nonlinear Schrödinger equations, respectively [16]. It is interesting that proper exact reductions of the Korteweg-deVries (KdV) equation lead to P I and P II [17]. iii) There exists a deep connection between equations with the Painleve property and monodrony preserving deformations. Actually aspects of this connection were already discussed at the beginning of the century (see below). Recently monodrony preserving deformations, about regular and irregular singular points of linear ODE's, have been discussed by Sato et al. [18] and Ueno [19], Flaschka and Newell [20], and Jimbo et al. [21], respectively.

In this paper we consider the initial value problem of P II,

$$
q_{t t}=2 q^{3}+t q-v,
$$

where $v$ is a constant parameter. In this respect we note: i) Ablowitz and Segur [16] characterized a non-elementary one-parameter family of solutions of (1.1) with $v=0$, through a Gel'fand-Levitan-Marchenko integral equation of the Fredholm type. ii) Flaschka and Newell [20] considered (1.1) as a deformation equation of the following system of linear ODE's

$$
\omega_{\zeta}(\zeta ; t)=\left(\begin{array}{cc}
-i\left(4 \zeta^{2}+t+2 q^{2}\right) & \left(4 \zeta q+2 i q_{t}+\frac{v}{\zeta}\right) \\
\left(4 \zeta q-2 i q_{t}+\frac{v}{\zeta}\right) & i\left(4 \zeta^{2}+t+2 q^{2}\right)
\end{array}\right) \omega(\zeta ; t) .
$$

Solving the initial value problem of (1.1) amounts to solving an "inverse problem" for $\omega(\zeta ; t)$, i.e. given appropriate monodromy data reconstruct $\omega(\zeta ; t)$. Obtaining this monodromy data is classical and corresponds to the direct problem in IST. The solution of the inverse problem is given in [20] in terms of what the authors of [20] call a system of singular integral equations (see Appendix B). iii) Jimbo et al. [21] considered PII, as part of a larger program of study of monodromy preserving deformations for a first order matrix system of ODE's having regular or irregular singularities of arbitrary rank. In their work the inverse problem is solved in terms of an infinite formal series uniquely determined in terms of certain monodromy data. iv) In [22] we characterized a three-parameter family of solutions of

$$
U_{t t t}+6 U U_{t}-\left(2 U+t U_{t}\right)=0
$$

using a linear singular integral equation. This equation was mapped to a matrix system of Fredholm integral equations. However, the question of whether or not this is the general solution of (1.3) was left open. Equation (1.3) is related [23] to P II through a one to one transformation [17]. It turns out that the solution of (1.3) obtained in [22] corresponds only to a specific range of $v$.

In this paper we consider the inverse problem for P II in terms of a $\mathrm{RH}$ boundary value problem. This $\mathrm{RH}$ problem is defined with respect to six semi-rays 
separated by an angle of $\pi / 3$ from each other. Solving the inverse problem is tantamount to constructing a sectionally analytic function $\psi_{v}(z ; t)$ with given "jumps" across the above semi-rays. These jumps are uniquely defined in terms of the "stokes multipliers" (part of the monodromy data). A necessary condition for the solvability of the above $\mathrm{RH}$ problem is that a certain product condition must be satisfied at the origin. This condition imposes a constraint between the stokes multipliers, the so-called connection matrix, and the monodromy matrix at the origin. i) In the case that $0 \leqq v<1$ the above $\mathrm{RH}$ problem is a matrix discontinuous RH problem on a complicated contour (six semi-rays intersecting at the origin). Through a series of transformations we map this problem to three simple $\mathrm{RH}$ problems, each defined on two rays (which provide the continuation of each other). Two of these RH problems are continuous, while the third has a discontinuity at the origin. Each of those $\mathrm{RH}$ problems, using standard methods is then reduced to a system of two Fredholm integral equations of the second type. The question of existence and uniqueness of these Fredholm equations can be addressed using standard analysis. ii) In the case that $v \geqq 1$ the above RH problem has a non-integrable singularity at the origin. One has then to suitably subtract the "singular part" before applying the method of i). In this process one discovers a relationship between $\psi_{v}(z ; t)$ and $\psi_{1-v}(z ; t)$. This relationship easily yields the known transformation between $q_{v}(t)$ and $q_{1-\nu}(t)$. Hence, using induction one only needs to consider the range $0 \leqq v<1$.

The correspondence of our integral equations with those given in [20] is discussed in Appendix B; here we only mention that in solving the inverse problem we have abandoned the approach of [20], which we find ineffective.

Before concentrating on the main results of this paper, we shall first discuss briefly the connection between the method of solution of the initial value problem of P II used here, and the IST method as applied to problems in $1+1$ (i.e. one space and one time) and 2+1 [24-26]. We hope that from this discussion it will become clear that there exists a unified approach to initial value problems in $1,1+1$, and $2+1$ dimensions: Solving the initial value problem of some equation for $q(t)$ or $q(x, t)$ or $q(x, y, t)$ is equivalent to solving an inverse problem for some related eigenfunction $\psi(z ; t)$ or $\psi(z ; x, t)$ or $\psi(z ; x, y, t)$. The inverse problem generically takes the form of a $\mathrm{RH}$ problem for equations in $1,1+1$, and in general the form of a $\bar{\partial}(\mathrm{DBAR})$ problem for equations in $2+1$ (a DBAR problem is a generalization of a $\mathrm{RH}$ problem). To define the relevant $\mathrm{RH}$ or $\mathrm{DBAR}$ problems one needs to study the analyticity properties of $\psi$ with respect to $z$. Furthermore these problems are uniquely defined in terms of certain asymptotic data of the underlying linear system satisfied by $\psi$ (monodromy data in the case of equations in 1 dimension and scattering data in the case of equations in $1+1$ and $2+1$ ).

\section{The General Framework}

\section{A. RH Problems and IST}

Let $C$ be a simple, smooth, closed contour dividing the complex $z$-plane into two regions $D^{+}$and $D^{-}$(the positive direction of $C$ will be taken as that for which $D^{+}$ is on the left). 
Fig. 1

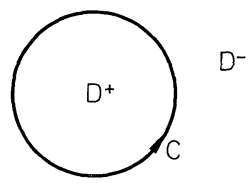

A function $\phi(z)$ defined in the entire plane, except for points on $C$ which will be called sectionally holomorphic if: i) the function $\phi(z)$ is holomorphic in each of the regions $D^{+}$and $D^{-}$except, perhaps, at $z=\infty$; ii) the function $\phi(z)$ is sectionally continuous with respect to $C$, approaching the definite limiting values $\phi^{+}(\zeta), \phi^{-}(\zeta)$ as $z$ approaches a point $\zeta$ on $C$ from $D^{+}$, or $D^{-}$, respectively. The classical homogeneous $\mathrm{RH}$ problem is defined as follows [27]. Given a contour $C$, and a function $G(\zeta)$ which is Hölder on $C$ and $\operatorname{det} G(t) \neq 0$ on $C$, find a sectionally holomorphic function $\phi(z)$, with finite degree at $\infty$, such that

$$
\phi^{+}(\zeta)=G(\zeta) \phi^{-}(\zeta), \quad \text { on } C,
$$

where $\phi^{ \pm}(\zeta)$ are the boundary values of $\phi(z)$ on $C$. If $G(\zeta)$ is scalar, (2.1) is solvable in closed form. If $G(\zeta)$ is a matrix valued function, then (2.1) is in general solvable in terms of a system of Fredholm integral equations. Various generalizations of the above $\mathrm{RH}$ problem are possible. For example: i) The contour $C$ may be replaced by a union of intersecting contours. ii) $G(\zeta)$ may have simple discontinuities at a finite number of points; in this case one allows $\phi(z)$ to have integrable singularities in the neighborhood of these points. iii) $\mathrm{RH}$ problems may be considered in other than Hölder spaces (e.g. [28]): iv) One may consider inhomogeneous RH problems $\phi^{+}(\zeta)=G(\zeta) \phi^{-}(\zeta)+F(\zeta)$ on $C$.

The connection between $\mathrm{RH}$ problems and IST in $1+1$ results from the following: A necessary condition for a given nonlinear equation for $q(x, t)$ to be solvable via IST is that $q(x, t)$ is related to some linear eigenvalue problem. For example for the $\mathrm{MKdV}$ equation [16]

$$
\psi_{x}(\zeta, x, t)=\zeta\left(\begin{array}{cc}
-i & 0 \\
0 & i
\end{array}\right) \psi(\zeta, x, t)+\left(\begin{array}{cc}
0 & q(x, t) \\
q(x, t) & 0
\end{array}\right) \psi(\zeta, x, t) .
$$

To solve the initial value problem for $q(x, t)$, one first formulates an inverse problem for $\psi(z ; x, t)$ : Given appropriate scattering data reconstruct $\psi$. The solution of this inverse problem is obtained via a $\mathrm{RH}$ problem for the sectionally meromorphic function (with respect to $z$ ) $\psi$. To define this $\mathrm{RH}$ problem one needs to use (2.2) to study the analytic properties of $\psi$ with respect to $z$. It is evident that one may pose the inverse problem for any function $q(x)$. However, this result can be used to solve the initial value problem of $q(x, t)$, only if $q$ evolves in such a way in $t$ that the scattering data is known for all $t$. This yields only a specific class of evolution equations solvable via (2.2) (sometimes referred to in the literature as the isospectral class).

The situation is conceptually also similar in $2+1$. Equation (2.2) is now replaced by a linear PDE for $\psi$ in $x$ and $y$. Again one formulates an inverse problem for $\psi(z ; x, y, t)$. However, the solution of this inverse problem is now obtained via a DBAR problem (this results from the fact that the analyticity structure of $\psi$ with respect to $z$ is now more complicated). 


\section{B. Monodromy Problems}

It is remarkable that the first RH problem was formulated in connection with an inverse problem: Consider the following system of linear ODE's

$$
\frac{d \psi(\zeta)}{d \zeta}=\sum_{i=1}^{n} \frac{A_{i}}{\zeta-a_{i}} \psi(\zeta)
$$

where $a_{i}$ are complex constant scalars and $A_{i}$ constant $m \times m$ matrices. It is clear that $\psi(z)$ is a multivalued analytic function with branch points at $a_{i}, i=1, \ldots, n$; in the neighborhood of $a_{i}, \psi(z) \sim H_{i}(z)\left(z-a_{i}\right)^{-L_{i}}$, where $H_{i}$ are holomorphic and invertible near $a_{i}$ and $L_{i}, i=1, \ldots, n$ are constant matrices, related to the monodromy matrices $M_{i} \doteqdot \exp \left[2 \pi i L_{i}\right]$. In connection with (2.3), Riemann [29] formulated the following inverse problem, which is known as the "Riemann monodromy" (RM) problem: Given branch points $a_{1}, \ldots, a_{n}$ and matrices $M_{1}, \ldots, M_{n}$, find $\psi$. This problem was reduced by Riemann to a discontinuous matrix $\mathrm{RH}$ problem on the contour joining the branch points; the jumps across this contour are uniquely determined in terms of the monodromy matrices. The RH problem has been considered by: i) Hilbert [30], Birkhoff [31], and extensively by Plemelj [32] using its equivalence with a RH problem. ii) By Lappo Danilevskij in terms of hyperlogarithe functions [33]. iii) By Sato et al. [18] using the equivalence between the RM problem and the so-called Schlesinger equations (see below), and employing classical operators of field theory. See also [34].

The use of the above inverse problem for solving certain nonlinear equations is similar to the one considered in $A$ above: Suppose that $A_{i}$ depend on certain parameters $t=\left(t_{1}, \ldots, t_{N}\right)$. Can one impose such an evolution of $A$ with respect to $t$, that the underlying monodromy data and known for all $t$ ? The answer is affirmative provided that $A_{i}$ satisfy a system of nonlinear equations known as the Schlesinger system.

The above considerations have been extended to systems of linear ODE's with irregular singular points [31] and [19-21]. Given such a system there exist two fundamental questions : i) Find all nonlinear equations (Schlesinger system) which are related to this linear system of ODE's (i.e. find all its isomonodromic deformations). This question has been exhaustively answered in [21]. ii) Solve the relevant inverse problem. This question has been considered in general in $[21,35]$ using infinite series, and concretely for the inverse problems associated with P II and P III in [20]. Here we solve the inverse problem associated with (1.2) by using a $\mathrm{RH}$ problem as well as a sequence of appropriate transformations.

\section{The Direct Problem}

P II can be written as the compatibility condition of (1.2) and

$$
\omega_{t}=\zeta\left(\begin{array}{cc}
-i & 0 \\
0 & i
\end{array}\right) \omega(\zeta ; t)+\left(\begin{array}{cc}
0 & q(t) \\
q(t) & 0
\end{array}\right) \omega(\zeta ; t) .
$$

Let $W(z)$ be a fundamental solution matrix of (1.2) (for simplicity of notation we suppress the $t$-dependence). The essence of the direct problem is to establish the 
analytic structure of $W$ with respect to $z$, in the entire complex $z$-plane. It should be pointed out that, in contrast to the analogous problem in IST for $1+1$ and $2+1$, this task here is straightforward: Equation (1.2) is a linear ODE in $z$, therefore its analytic structure is completely determined by its singular points. Equation (1.2) has a regular singular point at the origin (if $v \neq 0$ ) and an irregular singular point at infinity. In order that one is able to formulate a $\mathrm{RH}$ problem one must know the behavior of $W(z)$ as $z \rightarrow \infty$. Suppose that $W(z) \rightarrow \tilde{W}$ as $z \rightarrow \infty$ in $S_{1}$ (some sector of the complex $z$-plane). Then, according to the Stokes phenomenon, $W(z) \rightarrow \tilde{W} G$, where $G$ is some constant matrix containing the so-called Stokes multipliers. Alternatively one may choose different fundamental matrices in different sectors by the requirement that all have the same asymptotic behavior as $z \rightarrow \infty$. In connection with (1.2), there exist six sectors; let $W \doteqdot W_{i}$ if $z$ in $S_{i}$. Then (details of the direct problem can be found in [20])

$$
\begin{aligned}
W(z) \doteqdot W_{i}(z), & z \text { in } S_{i} \\
W_{2}(z)=W_{1}(z) G_{1}, & \frac{\pi}{3} \leqq \arg z<\frac{2 \pi}{3}, \\
W_{3}(z)=W_{2}(z) G_{2}, & \frac{2 \pi}{3} \leqq \arg z<\pi, \\
W_{4}(z)=W_{3}(z) G_{3}, & \pi \leqq \arg z<\frac{4 \pi}{3}, \\
W_{5}(z)=W_{4}(z) G_{4}, & \frac{4 \pi}{3} \leqq \arg z<\frac{5 \pi}{3}, \\
W_{6}(z)=W_{5}(z) G_{5}, & \frac{5 \pi}{3} \leqq \arg z<2 \pi \\
W_{1}\left(z e^{2 i \pi}\right)=W_{6}(z) G_{6}, & 2 \pi \leqq \arg z<\frac{7 \pi}{3},
\end{aligned}
$$

where

$$
\begin{array}{rlrl}
G_{1} & =\left(\begin{array}{ll}
1 & 0 \\
a & 1
\end{array}\right), & G_{2}=\left(\begin{array}{ll}
1 & b \\
0 & 1
\end{array}\right), & G_{3}=\left(\begin{array}{ll}
1 & 0 \\
c & 1
\end{array}\right), \\
G_{4}=\left(\begin{array}{ll}
1 & a \\
0 & 1
\end{array}\right), & G_{5}=\left(\begin{array}{ll}
1 & 0 \\
b & 1
\end{array}\right), & G_{6}=\left(\begin{array}{ll}
1 & c \\
0 & 1
\end{array}\right) .
\end{array}
$$

As $z \rightarrow \infty$ in $S_{1}, W_{1}(z)$ asymptotically tends to

$$
W_{1}(z)\left(\begin{array}{cc}
e^{\theta(z, t)} & 0 \\
0 & e^{-\theta(z, t)}
\end{array}\right) \sim I+\frac{1}{z}\left(\begin{array}{cc}
-\frac{i}{2} K(t) & -\frac{i}{2} q(t) \\
\frac{i}{2} q(t) & \frac{i}{2} K(t)
\end{array}\right)+O\left(\frac{1}{z^{2}}\right),
$$

where $I$ is the unit $2 \times 2$ matrix and

$$
\theta(z, t) \doteqdot \frac{4 i z^{3}}{3}+i z t, K(t) \doteqdot q_{t}^{2}-t q^{2}-q^{4}+2 v q .
$$


As $z \rightarrow 0$, two linearly independent solutions of (1.2) are given by [for $v \neq(2 n+1) / 2$ ]

$$
\Phi(z)=\left(\frac{e^{-u(t)}}{\sqrt{2} z^{v}}\left\{\left(\begin{array}{c}
1 \\
-1
\end{array}\right)-\frac{i z}{1-2 v} \Lambda(t)\left(\begin{array}{l}
1 \\
1
\end{array}\right)+O\left(z^{2}\right)\right\}, \frac{z^{v} e^{u(t)}}{\sqrt{2}}\left\{\left(\begin{array}{l}
1 \\
1
\end{array}\right)+O(z)\right\}\right),
$$

where $\Lambda(t) \doteqdot 2 q_{t}+2 q^{2}+t$ and the factors $\exp [ \pm u(t)], u_{t} \doteqdot q$ have been introduced in order that (3.6) satisfies (3.1). If $v=(2 n+1) / 2$, Eq. (3.6) must be appropriately modified. This leads to the following monodromy matrix $J$ associated with $z=0$ $\Phi\left(z e^{2 i \pi}\right)=\Phi(z) J ; J=\left(\begin{array}{cc}e^{-2 \pi i v} & 0 \\ 0 & e^{2 \pi i v}\end{array}\right), v \neq \frac{2 n+1}{2}, J=-\left(\begin{array}{cc}1 & 0 \\ 2 \pi \hat{\Lambda} & 1\end{array}\right), v=\frac{2 n+1}{2}$,

where $\hat{\Lambda}$ is the coefficient of $\zeta^{(2 v-1) / 2}$ in the series (3.6), and it is shown in [20] that it is independent of $t$. The matrices $W_{1}(z)$ and $\Phi(z)$ are related through the connection matrix $E$

$$
W_{1}(z)=\Phi(z) E, E \doteqdot\left(\begin{array}{ll}
\alpha & \beta \\
\gamma & \delta
\end{array}\right), \operatorname{det} E=1
$$

Flashka and Newell [20], using (3.2) and a certain symmetry relationship of $\Phi$, have established the following relationships between $G_{i}, J$, and $E$ :

$$
\begin{gathered}
1+a b=2 \alpha \gamma \cos v \pi-\pi \hat{\Lambda} \alpha^{2} e^{-v \pi i}, \quad 1+b c=-2 \beta \delta \cos v \pi+\pi \hat{\Lambda} \beta^{2} e^{-v \pi i}, \\
b=-\alpha \delta e^{v \pi i}-\beta \gamma e^{-v \pi i}+\pi \hat{\Lambda} \alpha \beta e^{-v \pi i}, \alpha+b+c+a b c=-2 i \sin v \pi .
\end{gathered}
$$

\section{The Inverse Problem}

This is the main section of this paper. It is concerned with the formulation and solution of the inverse problem associated with (1.2), i.e. given suitable monodromy data reconstruct $W(z)$. It is convenient to work with

$$
\Psi_{j}(z) \doteqdot W_{j}(z) Y^{-1}, 1 \leqq j \leqq 6 ; Y \doteqdot \operatorname{Diag}\left(e^{-\theta}, e^{\theta}\right) .
$$

Then (3.2) imply the following jump conditions for the function $\Psi(z)$

$$
\Psi_{j+1}(\zeta)=\Psi_{j}(\zeta) g_{j}(\zeta), 1 \leqq j \leqq 6 ; \Psi_{7}(\zeta) \doteqdot \Psi_{1}\left(\zeta e^{2 i \pi}\right), \zeta \quad \text { on } \quad C
$$

where $C=C_{1}+\ldots+C_{6}, g_{j}(\zeta) \doteqdot Y G_{j} Y^{-1}$, i.e.

$$
\begin{array}{cc}
g_{1}=\left(\begin{array}{cc}
1 & 0 \\
a e^{2 \theta} & 1
\end{array}\right), g_{2} & =\left(\begin{array}{cc}
1 & b e^{-2 \theta} \\
0 & 1
\end{array}\right), \quad g_{3}=\left(\begin{array}{cc}
1 & 0 \\
c e^{2 \theta} & 1
\end{array}\right), \quad g_{4}=\left(\begin{array}{cc}
1 & a e^{-2 \theta} \\
0 & 1
\end{array}\right), \\
g_{5} & =\left(\begin{array}{cc}
1 & 0 \\
b e^{2 \theta} & 1
\end{array}\right), \quad g_{6}=\left(\begin{array}{cc}
1 & c e^{-2 \theta} \\
0 & 1
\end{array}\right) .
\end{array}
$$

Also

$$
\Psi(z) \rightarrow I \quad \text { as } \quad z \rightarrow \infty, \quad \Psi_{1}(z) \sim \Phi(z) E \quad \text { as } \quad z \rightarrow 0 .
$$

Equations (4.2)-(4.4) define a singular, discontinuous, homogeneous RH problem for the sectionally analytic matrix-valued function $\Psi(z)$ : The function $\Psi(z)$ has different representations $\Psi_{1}(z), \ldots, \Psi_{6}(z)$ in the different sectors $S_{1}, \ldots, S_{6}$. The 
Fig. 2

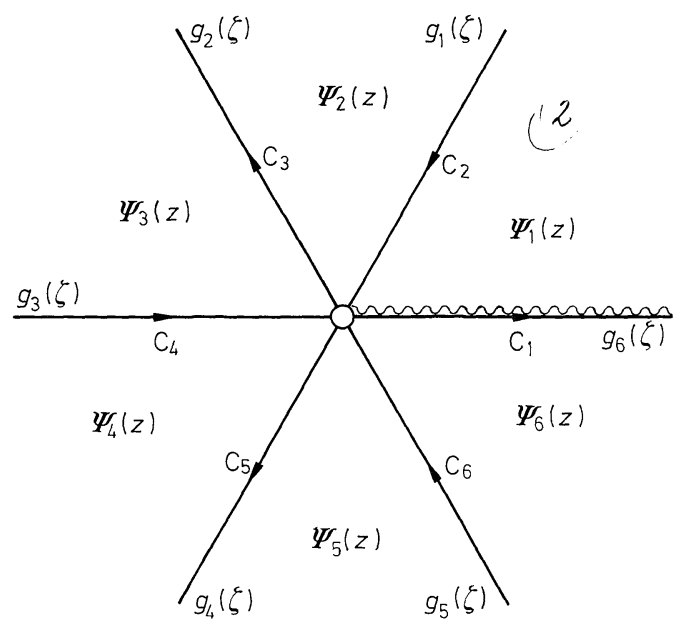

boundary values of these different representations are related through Eqs. (4.2). Furthermore, $\Psi_{j}(z)$ is analytic for $z$ in $S_{j}$ and $\Psi_{j}(z) \rightarrow I$ as $z \rightarrow \infty$ in $S_{j}$. The above $\mathrm{RH}$ is discontinuous because $g_{6}(0) \neq g_{3}(0), g_{1}(0) \neq g_{4}(0), g_{2}(0) \neq g_{5}(0)$. It is singular because at the origin $\Psi(z)$ possesses a non-integrable singularity, unless $0 \leqq v<1$ (note that $C_{1}$ is the contour from $\varepsilon$ to $\infty$, similarly for $C_{j}, 1<j \leqq 6$ ).

A. $0 \leqq v<1$

We first consider the above $\mathrm{RH}$ problem in the case that it is regular, i.e. $0 \leqq v<1$. It will turn out that the case of a general $v$ can be reduced to the case $0 \leqq v<1$.

Theorem 1. Consider the following matrix, discontinuous, homogeneous $R H$ problem along the six rays $C_{j}, 1 \leqq j \leqq 6$, intersecting at the origin (see Fig. 2): Determine the sectionally analytic function $\Psi(z), \Psi(z)=\Psi_{j}(z)$ if $z$ in $S_{j}$, from the following conditions :

ג) $\Psi_{j}(\zeta)$ satisfy the jump conditions (4.2) for $\zeta$ on $C$.

B) $\Psi(z) \rightarrow I+O\left(\frac{1}{z}\right)$ as $z \rightarrow \infty$.

$\gamma) \Psi(z)$ has at most an integrable singularity at the origin.

ס) $\Psi_{1}(z)$ possesses the following monodromy matrix at the origin.

$$
\Psi_{1}\left(z e^{2 i \pi}\right)=\Psi_{1}(z) \hat{J}, z \rightarrow 0 ; \hat{J}=E^{-1} J E,
$$

where $J$ is defined by (3.7) $(0 \leqq v<1)$. Then

i) A necessary condition for the solvability of the above $\mathrm{RH}$ problem is

$$
\hat{J} G_{1} G_{2} G_{3} G_{4} G_{5} G_{6}=I \text {. }
$$

ii) If (4.6) is satisfied then the solution of the above $R H$ problem can be obtained as follows: First solve the following RH problems

a)

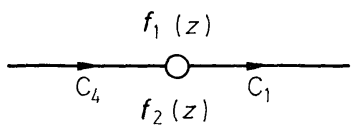

Fig. 3a 


$$
\begin{aligned}
& f_{1}(\zeta)=f_{2}(\zeta) g_{a}(\zeta), \quad \zeta \quad \text { on } C_{1}+C_{4} \\
& f_{1}(\zeta) \rightarrow I \quad \text { as } z \rightarrow \infty \quad \text { in } \quad S_{1}+S_{2}+S_{3} ; \\
& g_{a}(\zeta)=\left[\begin{array}{lllll}
g_{6}(\zeta), \\
A G_{1} G_{2}\left(G_{1} G_{2} g_{3}(\zeta)\right)^{-1} A^{-1}, & \zeta & \text { on } & C_{4}
\end{array},\right.
\end{aligned}
$$

where $A$ is defined by

$$
A G_{1} G_{2}\left(G_{1} G_{2} G_{3}\right)^{-1} A^{-1}=G_{6}
$$

b)

\section{Fig. 3b}

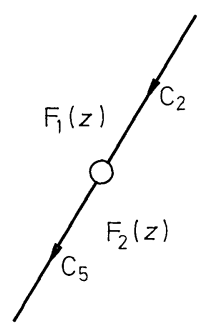

$F_{2}(\zeta)=F_{1}(\zeta) g_{b}(\zeta), \quad \zeta \quad$ on $\quad C_{2}+C_{5}$;

$F_{2}(z) \rightarrow I, \quad z \rightarrow \infty \quad$ in $S_{5}+S_{6}+S_{1}$

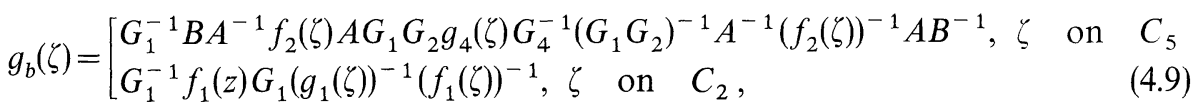

where $B$ is defined by

$$
B G_{1} G_{2} G_{4}^{-1}\left(G_{1} G_{2}\right)^{-1} B^{-1}=G_{1} \text {. }
$$

c)

Fig. 3c

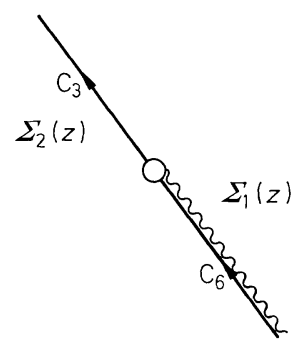

$\Sigma_{2}(\zeta)=\Sigma_{1}(\zeta) g_{c}(\zeta), \quad \zeta \quad$ on $\quad C_{3}+C_{6}$

$\Sigma_{1}(z) \rightarrow I, \quad z \rightarrow \infty \quad$ in $S_{6}+S_{1}+S_{2}$

$g_{c}(\zeta)=\left[\begin{array}{l}F_{1}(\zeta) G_{1}^{-1} f_{1}(\zeta) G_{1} g_{2}(\zeta)\left(G_{1} G_{2}\right)^{-1} A^{-1}\left(f_{1}(\zeta)\right)^{-1} A B^{-1} G_{1}\left(F_{1}(\zeta)\right)^{-1}, \zeta \text { on } C_{3} \\ F_{2}(\zeta) f_{2}(\zeta)\left(g_{5}(\zeta)\right)^{-1} G_{4}^{-1}\left(G_{1} G_{2}\right)^{-1} A^{-1}\left(f_{2}(\zeta)\right)^{-1} A B^{-1}\left(F_{2}(\zeta)\right)^{-1}, \zeta \text { on } C_{6} .\end{array}\right.$ 
Then define:

a) $\chi_{16}(z), \chi_{2}(z), \chi_{34}(z), \chi_{5}(z)$, for $z$ in $S_{1}+S_{6}, S_{2}, S_{2}+S_{4}, S_{5}$, respectively, as follows

$$
\begin{aligned}
\chi_{2}(z) & =\Sigma_{1}(z) F_{1}(z) G_{1}^{-1}, & \chi_{5}(z) & =\Sigma_{2}(z) F_{2}(z) B A^{-1}, \\
\chi_{34}(z) & =\Sigma_{2}(z) F_{1}(z) G_{1}^{-1} B A^{-1}, & \chi_{16}(z) & =\Sigma_{1}(z) F_{2}(z) .
\end{aligned}
$$

b) $\phi_{1}(z), \ldots, \phi_{6}(z)$ for $z$ in $S_{1}, \ldots, S_{6}$, respectively, as follows

$$
\begin{array}{ll}
\phi_{1}(z)=\chi_{16}(z) f_{1}(z), & \phi_{4}(z)=\chi_{34}(z) f_{2}(z) A\left(G_{1} G_{2}\right)\left(G_{1} G_{2} G_{3}\right)^{-1}, \\
\phi_{2}(z)=\chi_{2}(z) f_{1}(z), & \phi_{5}(z)=\chi_{5}(z) f_{2}(z) A\left(G_{1} G_{2}\right)\left(G_{1} G_{2} G_{3}\right)^{-1}, \\
\phi_{3}(z)=\chi_{34}(z) f_{1}(z) A, & \phi_{6}(z)=\chi_{16}(z) f_{2}(z)\left(G_{1} G_{2} G_{3} G_{4} G_{5}\right)^{-1} .
\end{array}
$$

c) $\psi_{1}(z), \psi_{2}(z), \ldots, \psi_{6}(z)$ for $z$ in $S_{1}, \ldots, S_{6}$, respectively, as follows

$$
\begin{array}{ll}
\psi_{1}(z)=\phi_{1}(z), & \psi_{4}(z)=\phi_{4}(z) G_{1} G_{2} G_{3}, \\
\psi_{2}(z)=\phi_{2}(z) G_{1}, & \psi_{5}(z)=\phi_{5}(z) G_{1} G_{2} G_{3} G_{4}, \\
\psi_{3}(z)=\phi_{3}(z) G_{1} G_{2}, & \psi_{6}(z)=\phi_{6}(z) G_{1} G_{2} G_{3} G_{4} G_{5} .
\end{array}
$$

Remarks. a) The RH problem (4.7) is continuous both at the origin and at infinity. Actually

$$
g_{a}(0+)=g_{a}(0-)=G_{6} ; \quad g_{a}(\infty)=g_{a}(-\infty)=I .
$$

b) Similarly for the $\mathrm{RH}$ problem (4.9):

$$
g_{b}(0+)=g_{b}(0-)=G_{1}^{-1} ; \quad g_{b}(\infty)=g_{b}(-\infty)=I,
$$

since $f \rightarrow I$ as $z \rightarrow \infty$ [using $g_{a}( \pm \infty)=I$ ].

c) The RH problem (4.11) is continuous at infinity but discontinuous at the origin:

$$
g_{c}(\infty)=g_{c}(-\infty)=\left(G_{1} G_{2}\right)^{-1} B^{-1} G_{1},
$$

since $F \rightarrow I$ as $z \rightarrow \infty$ [using $g_{b}( \pm \infty)=I$ ]. Furthermore, the discontinuity at the origin is related to the monodromy matrix $M$ of $\Sigma$, which is in turn related to the monodromy matrix $\hat{J}$ of $\psi$ : let $M$ be the monodromy matrix of $\Sigma_{1}$, i.e.

$$
\Sigma_{1}\left(z e^{2 i \pi}\right)=\Sigma_{1}(z) M .
$$

Then equations $\psi_{1}(z)=\phi_{1}(z), \phi_{1}(z)=\chi_{16}(z) f_{1}(z), \chi_{16}(z)=\Sigma_{1}(z) F_{2}(z)$ imply that

$$
M=F_{20} f_{10} \hat{J}\left(F_{20} f_{10}\right)^{-1},
$$

where $f_{10}, F_{20}$, denote $f_{1}$ and $F_{2}$ at the origin. The origin condition of (4.11) implies

$$
M g_{c}(0+) g_{c}^{-1}(0-)=I \text {. }
$$

It is easily seen [using $A_{3}^{-1} G_{6}^{-1} A_{3}=G_{1} G_{2} G_{3}\left(G_{1} G_{2}\right)^{-1}$ and $F_{20}=F_{10} G_{1}^{-1}$, $\left.f_{10}=f_{20} G_{6}\right]$ that Eqs. (4.19), (4.20) are consistent.

d) The matrices $G_{1} G_{2} G_{3}\left(G_{1} G_{2}\right)^{-1}$ and $G_{1} G_{2} G_{4}\left(G_{1} G_{2}\right)^{-1}$ are non-unit matrices with one as a double eigenvalue. Hence the matrices $A$ and $B$ exist.

e) Equation (4.6) is valid iff $a+b+c+a b c= \pm 2 i \sin v \pi$, see Appendix A. 
Proof. To derive i) note that in the neighborhood of the origin

$$
\psi_{2}(z) \sim \psi_{1}(z) G_{1}, \psi_{3}(z) \sim \psi_{1}(z) G_{1} G_{2}, \ldots, \psi_{6}(z) \sim \psi_{1}(z) G_{1} G_{2} G_{3} G_{4} G_{5} .
$$

Also across $C_{1}$

$$
\psi_{1}(\varepsilon) \sim \psi_{6}\left(\varepsilon e^{2 i \pi}\right) G_{6} \sim \psi_{1}\left(\varepsilon e^{2 i \pi}\right) G_{1} G_{2} G_{3} G_{4} G_{5} G_{6} .
$$

Hence using (4.5) and the fact that $\psi_{1}(\varepsilon)$ is non-singular we obtain (4.6).

Now we concentrate on ii) of Theorem 1 . The basic idea of the above result is to use suitable transformations to map the $\mathrm{RH}$ problem for $\psi$ to simpler $\mathrm{RH}$ problems. These transformations must be such that the new $\mathrm{RH}$ problems are consistent at infinity and at the origin. Consistency at the origin means that the product of the jump matrices at the origin equals the inverse of the monodromy matrix about the origin. Similarly, consistency at infinity means that the product of the jump matrices at infinity equals the identity. It is clear that the $\mathrm{RH}$ problem for $\psi$ is consistent at infinity, since each monodromy matrix tends to $I$ at infinity.

The proof of Theorem 1 follows from the following lemmas:

Lemma 1. Let $\phi(z)=\phi_{j}(z)$ if $z$ in $S_{j}$ and

$$
\psi_{j}(z)=\phi_{j}(z) \sigma_{j}, \quad 1 \leqq j \leqq 6,
$$

where

$$
\sigma_{1}=I, \sigma_{2}=G_{1}, \sigma_{3}=G_{1} G_{2}, \sigma_{4}=G_{1} G_{2} G_{3}, \sigma_{5}=G_{1} G_{2} G_{3} G_{4}, \sigma_{6}=G_{1} G_{2} G_{3} G_{4} G_{5} .
$$

Then the $R H$ for $\psi$ is equivalent with the following $R H$ problem for $\phi$ :

i)

where

$$
\phi_{2}(\zeta)=\phi_{1}(\zeta) \tilde{g}_{1}(\zeta) \quad \text { on } \quad C_{2}, \quad \phi_{3}(\zeta)=\phi_{2}(\zeta) \tilde{g}_{2}(\zeta)
$$

$$
\begin{aligned}
& \tilde{g}_{1}(\zeta)=g_{1}(\zeta) G_{1}^{-1}, \quad \tilde{g}_{2}(\zeta)=G_{1} g_{2}(\zeta)\left(G_{1} G_{2}\right)^{-1}, \quad \tilde{g}_{3}(\zeta)=G_{1} G_{2} g_{3}(\zeta)\left(G_{1} G_{2} G_{3}\right)^{-1}, \\
& \tilde{g}_{4}(\zeta)=G_{1} G_{2} G_{3} g_{4}(\zeta)\left(G_{1} G_{2} G_{3} G_{4}\right)^{-1} \text {, } \\
& \tilde{g}_{5}(\zeta)=G_{1} G_{2} G_{3} G_{4} g_{5}(\zeta)\left(G_{1} G_{2} G_{3} G_{4} G_{5}\right)^{-1} \text {, } \\
& g_{6}(\zeta)=G_{1} G_{2} G_{3} G_{4} G_{5} g_{6}(\zeta) \text {. }
\end{aligned}
$$

ii) $\phi_{1}(z) \rightarrow I$ as $z \rightarrow \infty$ in $S_{1}$.

iii) $\phi(z)$ has at most an integrable singularity at the origin.

iv) $\phi_{1}(z)$ has $\hat{J}$ as its monodromy matrix at the origin.

Furthermore, the $R H$ problem for $\phi$ is discontinuous only along the real z-axis, it is continuous at infinity, and it is consistent at the origin iff the RH problem for $\psi$ is consistent, i.e. iff (4.5) is valid.

Proof. Recall that the RH problem for $\psi$ is discontinuous along every contour $C_{j}$. We determine the constant matrices $\sigma_{j}$ from the requirement that the $\mathrm{RH}$ for $\phi(z)$ has a discontinuity only along the real $z$-axis. Using (4.23) in (4.2) we obtain

$$
\begin{gathered}
\phi_{2}(\zeta)=\phi_{1}(\zeta) \sigma_{1} g_{1}(\zeta) \sigma_{2}^{-1} \text { on } C_{2}, \\
\phi_{3}(\zeta)=\phi_{2}(\zeta) \sigma_{2} g_{2}(\zeta) \sigma_{3}^{-1}, \quad \text { on } C_{3}, \ldots, \phi_{1}(\zeta)=\phi_{6}(\zeta) \sigma_{6} g_{6}(\zeta) \sigma_{1}^{-1} \text { on } C_{1} \cdot(4
\end{gathered}
$$


Fig. 4

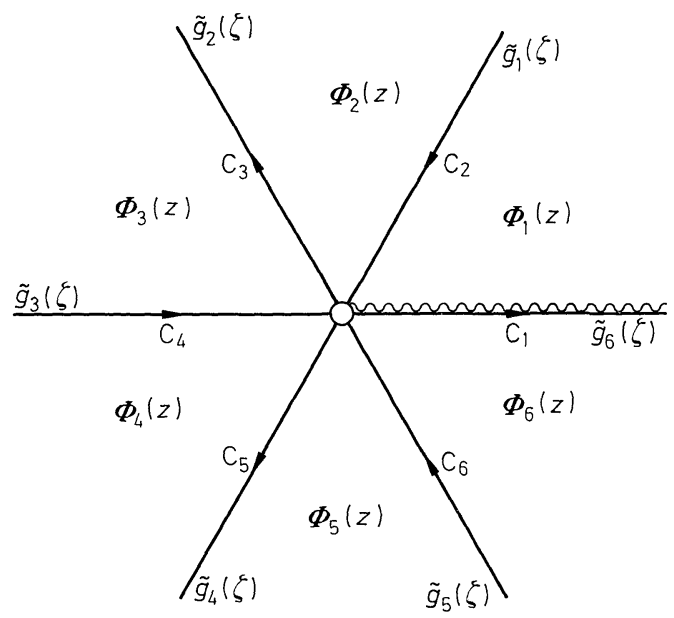

If in the neighborhood of the origin the matrices $\Psi$ and $\Phi$ are related by $\Psi(z)$ $=\Phi(z) A$, then their monodromy matrices $J_{\Psi}, J_{\Phi}$ are related by

$$
J_{\Phi}=A J_{\Psi} A^{-1} \text {. }
$$

Hence, Eqs. (4.23), (4.5) imply

$$
J_{\Phi}=\sigma_{1} \hat{J} \sigma_{1}^{-1} .
$$

The consistency condition at the origin for the $\mathrm{RH}$ for $\phi$ implies

$$
J_{\Phi}\left(\sigma_{1} g_{1} \sigma_{2}^{-1}\right)\left(\sigma_{2} g_{2} \sigma_{3}^{-1}\right) \ldots\left(\sigma_{6} g_{6} \sigma_{1}^{-1}\right)=I,
$$

which [using (4.28)] is satisfied iff (4.6) is valid. We choose $\sigma_{1}, \ldots, \sigma_{6}$ in such a way that the jumps across $C_{2}, \ldots, C_{6}$ in the neighborhood of the origin all equal $I$. This implies (4.24), where we normalize $\sigma_{1}$ to be $I$ so that $J_{\Phi}=\hat{J}$. Note that the product of $\tilde{g}$ 's at infinity equals $I$, which implies that the RH problem for $\phi$ is continuous at infinity. Q.E.D.

Remarks. The above RH problem has a simple interpretation from the monodromy point of view: $\phi_{1}(z), \ldots, \phi_{6}(z)$ are the representations of the same fundamental matrix solution $\phi(z)$ in the different sectors $S_{1}, \ldots, S_{6}$. This should be contrasted with the $\psi_{1}(z), \ldots, \psi_{6}(z)$, which are different fundamental matrix solutions (related to each other through the monodromy matrices). Of course, while $\psi_{2}(z) \rightarrow I$ as $z \rightarrow \infty$ in $S_{2}, \phi_{2}(z) \rightarrow G_{1}^{-1}$ as $z \rightarrow \infty$ in $S_{i}$; similarly $\phi_{3}(z), \ldots, \phi_{6}(z)$ tend to different constant matrices as $z \rightarrow \infty$.

The RH problem for $\phi(z)$ can be reduced to the three simpler RH problems (4.7), (4.9), (4.11). The idea is similar to the one used by Beals and Coifman [36] for a scalar, continuous $\mathrm{RH}$ problem.

We first reduce the RH for $\phi(z)$ to one for $\chi(z)$ and one for $f(z)$ :

Lemma 2. Let $\chi(z)=\chi_{16}(z)$ if $z$ in $S_{1}+S_{6}, \chi(z)=\chi_{2}(z)$ if $z$ in $S_{2}, \chi(z)=\chi_{34}(z)$ if $z$ in $S_{3}+S_{4}, \chi(z)=\chi_{5}(z)$ if $z$ in $S_{5}$. Also let $f(z)=f_{1}(z)$ if $z$ in $S_{1}+S_{2}+S_{3}, f(z)=f_{2}(z)$ 
Fig. 5
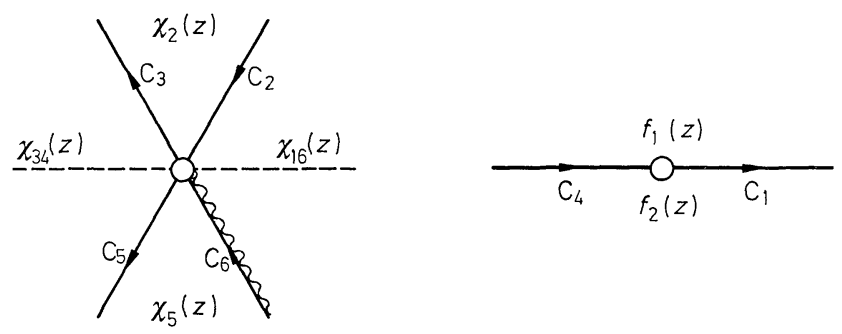

if $z$ in $S_{4}+S_{5}+S_{6}$. Relate $\chi(z)$ and $f(z)$ to $\phi(z)$ via

$$
\begin{array}{ll}
\phi_{1}(z)=\chi_{16}(z) f_{1}(z) A_{1}, & \phi_{4}(z)=\chi_{34}(z) f_{2}(z) B_{1}, \\
\phi_{2}(z)=\chi_{2}(z) f_{1}(z) A_{2}, & \phi_{5}(z)=\chi_{5}(z) f_{2}(z) B_{2}, \\
\phi_{3}(z)=\chi_{34}(z) f_{1}(z) A_{3}, & \phi_{6}(z)=\chi_{16}(z) f_{2}(z) B_{3},
\end{array}
$$

where the constant matrices $A_{i}, B_{i}$ satisfy

$$
\begin{aligned}
B_{1}= & A_{3} G_{1} G_{2}\left(G_{1} G_{2} G_{3}\right)^{-1}, \quad B_{3}=A_{1}\left(G_{1} G_{2} G_{3} G_{4} G_{5}\right)^{-1}, \\
& A_{1}^{-1} A_{3} G_{1} G_{2} G_{3}\left(G_{1} G_{2}\right)^{-1}\left(A_{1}^{-1} A_{3}\right)^{-1}=G_{6}^{-1} .
\end{aligned}
$$

Then

a) $f(z)$ satisfies a continuous, both at the origin and infinity, $R H$ problem:

$$
\begin{gathered}
f_{2}(\zeta)=f_{1}(\zeta) A_{3} \tilde{g}_{3}(\zeta) B_{1}^{-1}, \zeta \text { on } C_{4} ; \quad f_{1}(\zeta)=f_{2}(\zeta) B_{3} \tilde{g}_{6}(\zeta) A_{1}^{-1}, \zeta \text { on } C_{1} \text {, (4.31a) } \\
f_{1}(z) \rightarrow I \text { as } z \rightarrow \infty \text { in } S_{1}+S_{2}+S_{3} .
\end{gathered}
$$

b) $\chi(z)$ satisfies a $R H$ problem continuous at infinity and discontinuous but consistent at the origin:

$$
\begin{gathered}
\chi_{2}(\zeta)=\chi_{16}(\zeta) f_{1}(\zeta) A_{1} \tilde{g}_{1}(\zeta) A_{2}^{-1} f_{1}^{-1}(\zeta) \quad \text { on } \quad C_{2}, \\
\chi_{5}(\zeta)=\chi_{34}(\zeta) f_{2}(\zeta) B_{1} \tilde{g}_{4}(\zeta) B_{2}^{-1} f_{2}^{-1}(\zeta) \text { on } C_{5}, \\
\chi_{34}(\zeta)=\chi_{2}(\zeta) f_{1}(\zeta) A_{2} \tilde{g}_{2}(\zeta) A_{3}^{-1} f_{1}^{-1}(\zeta) \quad \text { on } C_{3}, \\
\chi_{16}(\zeta)=\chi_{5}(\zeta) f_{2}(\zeta) B_{2} \tilde{g}_{5}(\zeta) B_{3}^{-1} f_{2}^{-1}(\zeta) \quad \text { on } C_{6}, \\
\chi_{16}(z) \sim A_{1}^{-1} \quad \text { as } \quad z \rightarrow \infty, \quad J_{\chi_{16}}=f_{10} A_{1} \hat{J} A_{1}^{-1} f_{10}^{-1},
\end{gathered}
$$

where $J_{\chi_{16}}$ denotes the monodromy matrix of $\chi_{16}$ at the origin.

Proof. The transformations (4.29) imply (4.31a), (4.32a). The equation $\phi_{1}(z)$ $=\chi_{16}(z) f_{1}(z) A_{1}$ implies $(4.32 \mathrm{~b})$ provided that $f_{1}(z)$ tends to a constant value $f_{10}$ at the origin; this is indeed the case iff $f(z)$ satisfies a continuous $\mathrm{RH}$ problem, i.e. iff

$$
A_{3} B_{1}^{-1}=A_{1} \hat{J} B_{3}^{-1} \text {, }
$$

where we have used (4.31a), $\tilde{g}_{3}(0)=I, \tilde{g}_{6}(0)=\hat{J}^{-1}$.

Equations $\phi_{1}(z)=\chi_{16}(z) f_{1}(z) A_{1}, \phi_{6}(z)=\chi_{16}(z) f_{2}(z) B_{3}$, as $z \rightarrow \infty$ imply

$$
B_{3}=f_{2 \infty}^{-1} f_{1 \infty} A_{1}\left(G_{1} G_{2} G_{3} G_{4} G_{5}\right)^{-1} \text {, }
$$


where we have used that $\phi_{1} \rightarrow I, \phi_{6} \rightarrow\left(G_{1} G_{2} G_{3} G_{4} G_{5}\right)^{-1}$ as $z \rightarrow \infty$ [see (4.14)]. Similarly $\phi_{3}(z)=\chi_{34}(z) f_{1}(z) A_{3}, \phi_{4}(z)=\chi_{34}(z) f_{2}(z) B_{1}$ imply

$$
B_{1}=f_{2 \infty}^{-1} f_{1 \infty} A_{3} G_{1} G_{2}\left(G_{1} G_{2} G_{3}\right)^{-1} .
$$

Equations (4.33), (4.34) imply (4.30c). Using (4.34) the continuity at infinity of both $\mathrm{RH}$ problems for $\chi$ and $f$ is verified. Also using $f_{20}=f_{10} A_{3} B_{1}^{-1}$ it is easily seen that the product condition at the origin for (4.32a) is consistent with the formula for $J_{\chi_{16}}$ given by (4.32b). Equations (4.30a), (4.30b), (4.31b) follow normalizing $f_{1 \infty}=I$. Q.E.D.

Lemma 3. Let $\Sigma(z)=\Sigma_{1}(z)$ if $z$ in $S_{6}+S_{1}+S_{2}, \Sigma(z)=\Sigma_{2}(z)$ if $z$ in $S_{3}+S_{4}+S_{5} ; F(z)$ $=F_{1}(z)$ if $z$ in $S_{2}+S_{3}+S_{4}, F(z)=F_{2}(z)$ if $z$ in $S_{5}+S_{6}+S_{1}$. Relate $\Sigma$ and $F$ to $\chi$ via

$$
\begin{aligned}
\chi_{2}(z) & =\Sigma_{1}(z) F_{1}(z) a_{1}, \quad \chi_{5}(z)=\Sigma_{2}(z) F_{2}(z) b_{1}, \\
\chi_{34}(z) & =\Sigma_{2}(z) F_{1}(z) a_{2}, \quad \chi_{16}(z)=\Sigma_{1}(z) F_{2}(z) b_{2},
\end{aligned}
$$

where the constant matrices $a_{i}, b_{i}$ satisfy

$$
\begin{gathered}
b_{1}=a_{2} A_{3} G_{1} G_{2}\left(G_{4}\right)^{-1}\left(G_{1} G_{2}\right)^{-1} A_{3}^{-1}, \quad b_{2}=a_{1} A_{1} G_{1} A_{1}^{-1}, \\
B G_{1} G_{2} G_{4}\left(G_{1} G_{2}\right)^{-1} B^{-1}=G_{1}^{-1}, \quad B \doteqdot A_{1}^{-1} a_{1}^{-1} a_{2} A_{3} .
\end{gathered}
$$

Then if $\chi$ satisfies the $R H$ (4.32) with

it follows that:

$$
A_{2}=A_{1}, \quad B_{2}=B_{1}
$$

a) $F(z)$ satisfies a continuous, both at the origin and infinity $R H$ problem:

$$
\begin{aligned}
F_{1}(\zeta)= & F_{2}(\zeta) b_{2} f_{1}(\zeta) A_{1} \tilde{g}_{1}(\zeta) A_{1}^{-1} f_{1}^{-1}(\zeta) a_{1}^{-1}, \zeta \text { on } C_{2} \\
F_{2}(\zeta)= & F_{1}(\zeta) a_{2} f_{2}(\zeta) B_{1} \tilde{g}_{4}(\zeta) B_{1}^{-1} f_{2}^{-1}(\zeta) b_{1}^{-1}, \zeta \text { on } C_{5} \\
& F_{1}(z) \rightarrow I \quad \text { as } \quad z \rightarrow \infty \quad \text { in } \quad S_{2}+S_{3}+S_{4} .
\end{aligned}
$$

b) $\Sigma(z)$ satisfies a $R H$ problem continuous at infinity and discontinuous but consistent at the origin

$$
\begin{aligned}
& \Sigma_{2}(\zeta)= \Sigma_{1}(\zeta) F_{1}(\zeta) a_{1} f_{1}(\zeta) A_{1} \tilde{g}_{2}(\zeta) A_{3}^{-1} f_{1}^{-1}(\zeta) a_{2}^{-1} F_{1}^{-1}(\zeta), \zeta \text { on } C_{3} \\
& \Sigma_{1}(\zeta)=\Sigma_{2}(\zeta) F_{2}(\zeta) b_{1} f_{2}(\zeta) B_{1} \tilde{g}_{5}(\zeta) B_{3}^{-1} f_{2}^{-1}(\zeta) b_{2}^{-1} F_{2}^{-1}(\zeta), \zeta \text { on } C_{6} \\
& \Sigma_{1}(z) \rightarrow I \quad \text { as } \quad z \rightarrow \infty \quad \text { in } \quad S_{6}+S_{1}+S_{2}, \quad J_{\Sigma_{1}}=F_{20} b_{2} f_{10} A_{1} \hat{J}\left(F_{20} b_{2} f_{10} A_{1}\right)^{-1} .
\end{aligned}
$$

Proof. The transformations (4.35) imply (4.38a), (4.39a). The equation $\chi_{16}(z)$ $=\Sigma_{1}(z) F_{2}(z) b_{2}$ and the expression for $J_{\chi_{16}}$ given by (4.32b), imply the expression for $J_{\Sigma_{1}}$ provided that $F_{2}(z)$ tends to the constant value $F_{20}$ at the origin; this is the case iff $F(z)$ satisfies a continuous $\mathrm{RH}$ problem, i.e. iff

$$
b_{2} a_{1}^{-1}=b_{1} a_{2}^{-1} \text {. }
$$

Equations (4.35) and (4.34b) as $z \rightarrow \infty$ imply

$$
b_{1}=F_{2 \infty}^{-1} F_{1 \infty} a_{2} A_{3} G_{1} G_{2}\left(G_{1} G_{2} G_{3} G_{4}\right)^{-1} B_{2}^{-1}, \quad b_{2}=F_{2 \infty}^{-1} F_{1 \infty} a_{1} A_{2} G_{1} A_{1}^{-1},
$$




$$
\Sigma_{1 \infty}=A_{1}^{-1} b_{2}^{-1} F_{2 \infty}^{-1}, \quad \Sigma_{2 \infty}=\left(G_{1} G_{2} G_{3} G_{4}\right)^{-1} B_{2}^{-1} b_{1}^{-1} F_{2 \infty}^{-1} .
$$

Assuming (4.37) and normalizing $F(z)$ by $F_{1}(z) \rightarrow I$ as $z \rightarrow \infty$, Eqs. (4.40), (4.41a) imply (4.36). Using (4.41a) the continuity at infinity of both RH problems for $F$ and $\Sigma$ is verified. Also the product condition at the origin for the RH problem for $\Sigma$ is consistent with the formula for $J_{\Sigma_{1}}$ given above. Q.E.D.

Theorem 1 follows from Lemmas $1-3$ by choosing $A_{1}=I, a_{1}=G_{1}^{-1}, A_{3}=A$. This implies $b_{2}=I, b_{1}=B A^{-1}$.

Given a discontinuous problem on a simple contour, it is standard to map it to a continuous one. The basic idea was introduced by Gakhov [27i], regarding a scalar RH problem, and consists of introducing suitable auxiliary functions $\Omega^{ \pm}(z)$ such that their discontinuities cancel the discontinuity of the jump function appearing in the $\mathrm{RH}$ problem. The case of matrix discontinuous $\mathrm{RH}$ problems was considered by Vekua [27iii]. For completeness we present the relevant results as applied to the RH for $z$.

Lemma 4. Consider the RH problem for the function $\Sigma(z)$, i.e. Eqs. (4.11), (4.18), (4.19) and assume that $v \neq 1 / 2$ (see remark below). Define the sectionally holomorphic function $\Phi(z)$, such that $\Phi(z)=\Phi^{+}(z)$ if $z$ in $S_{3}+S_{4}+S_{5}, \Phi(z)=\Phi^{-}(z)$ if $z$ in $S_{6}+S_{1}+S_{2}$, via the transformations

$$
\Sigma_{2}(z)=\Phi^{+}(z) \Omega^{+}(z) D_{1}, \quad \Sigma_{1}(z)=\Phi^{-}(z) \Omega^{-}(z) D_{2} .
$$

In the above equations

$$
\begin{gathered}
D_{2} \doteqdot E\left(F_{20} f_{10}\right)^{-1}, \quad D_{1} \doteqdot\left(\Omega^{+}(0+)\right)^{-1} \Omega^{-}(0+) D_{2} g_{c}(0+), \\
\Omega^{+}(z)=\operatorname{Diag}\left[\left(\frac{z}{z-i}\right)^{-v},\left(\frac{z}{z-i}\right)^{v}\right], \quad \Omega^{-}(z)=\operatorname{Diag}\left[\left(\frac{z}{z+i}\right)^{-v},\left(\frac{z}{z+i}\right)^{v}\right],
\end{gathered}
$$

and the branch cuts of $\Omega^{+}, \Omega^{-}$are chosen in such a way that $\Omega^{+}, \Omega^{-}$are analytic in $S_{3}+S_{4}+S_{5}, S_{6}+S_{1}+S_{2}$, respectively. Then $\Phi(z)$ satisfies the continuous $R H$ problem.

$$
\begin{aligned}
\Phi^{+}(\zeta)= & \Phi^{-}(\zeta) \Omega^{-}(\zeta) D_{2} g_{c}(\zeta) D_{1}^{-1}\left(\Omega^{+}(\zeta)\right)^{-1}, \zeta \text { on } C_{3}+C_{6}, \\
& \Phi^{-}(z) \rightarrow D_{2}^{-1} \text { as } z \rightarrow \infty \text { in } S_{6}+S_{1}+S_{2},
\end{aligned}
$$

where $g_{c}(\zeta)$ is defined by (4.11).

Proof. Consider the functions

$$
\omega_{j}^{+}(z)=\left(\frac{z}{z-i}\right)^{\lambda_{j}}, \quad \omega_{j}^{-}(z)=\left(\frac{z}{z+i}\right)^{\lambda_{j}}, \quad j=1,2 .
$$

For the function $\omega_{1}^{+}(z)$, the branch cuts of $z^{\lambda_{1}}$ and $(z-i)^{-\lambda_{1}}$ are defined from the origin to $-i \infty$ and from $i$ to $-i \infty$, respectively. Thus if $z$ is on $C_{3}+C_{6}$ it follows that $\omega_{1}^{+}(0+) / \omega_{1}^{+}(0-)=\exp \left[-i \lambda_{1} \pi\right]$. Similarly $\omega_{1}^{-}(0+) / \omega_{1}^{-}(0-)=\exp \left[i \lambda_{1} \pi\right]$ Define the matrices

$$
\Omega^{ \pm}(z) \doteqdot \operatorname{Diag}\left(\omega_{1}^{ \pm}(z), \omega_{2}^{ \pm}(z)\right), \quad \Omega \doteqdot\left(\Omega^{-}\right)^{-1} \Omega^{+} .
$$


Then it follows that

$$
\Omega(0-)(\Omega(0+))^{-1}=\operatorname{Diag}\left(e^{2 i \lambda_{1} \pi}, e^{2 i \lambda_{2} \pi}\right) .
$$

Equations (4.42), (4.46) imply that

$$
\Phi^{+}(\zeta)=\Phi^{-}(\zeta) \Omega^{+}(\zeta)(\Omega(\zeta))^{-1} D_{2} g_{c}(\zeta) D_{1}^{-1}\left(\Omega^{+}(\zeta)\right)^{-1}
$$

Choose $D_{1}, D_{2}$ such that

$$
(\Omega(0+))^{-1} D_{2} g_{c}(0+) D_{1}^{-1}=(\Omega(0-))^{-1} D_{2} g_{c}(0-) D_{1}^{-1}=I,
$$

i.e. $D_{1}$ as defined by $(4.43 \mathrm{~b})$ and $D_{2}$ via

$$
D_{2} g_{c}(0-) g_{c}(0+)^{-1} D_{2}^{-1}=\operatorname{Diag}\left(e^{2 i \lambda_{1} \pi}, e^{2 i \lambda_{2} \pi}\right) .
$$

Hence, (4.43a) follows using

$$
g_{c}(0-) g_{c}(0+)^{-1}=M=F_{20} f_{10} \hat{J}\left(F_{20} f_{10}\right)^{-1}=F_{20} f_{10} E^{-1} J E\left(F_{20} f_{10}\right)^{-1},
$$

where we have made use of (4.5), (4.19), (4.20). Q.E.D.

Remark. The direct problem implies that $v=1 / 2$ is somewhat special, since then $J$ is not diagonal. It is interesting that from the $\mathrm{RH}$ point of view $v=1 / 2$ is also special: In this case $J$ has a double eigenvalue and one must modify the approach suggested in Lemma 4. The corresponding general theory can be found in [27iii].

From the above discussion it follows that the original discontinuous, complicated problem for the function $\Psi(z)$ is equivalent to the three continuous simple RH problems for the functions $f(z), F(z), \Phi(z)$. By taking appropriate "projections," i.e. by using Plemelj's formula [27], the above RH problems are immediately reduced to Fredholm integral equations.

Proposition 1. The original $R H$ problem of the function $\Psi(z)$ defined in Theorem 1 , through the transformation of Theorem 1 and Lemma 4 is equivalent to the following linear Fredholm integral equations:

$$
\begin{gathered}
f_{2}(\zeta)=I+\frac{P_{1}}{2 \pi i}\left(\int_{C_{4}+C_{1}} \frac{f_{2}(\tau)\left(g_{a}(\tau)-I\right) d \tau}{\tau-z}\right)(\zeta), \zeta \text { on } C_{4}+C_{1}, \\
F_{1}(\zeta)=I+\frac{P_{2}}{2 \pi i}\left(\int_{C_{2}+C_{5}} \frac{F_{1}(\tau)\left(g_{b}(\tau)-I\right) d \tau}{\tau-z}\right)(\zeta), \zeta \text { on } C_{2}+C_{5}, \\
\Phi^{-}(\zeta)=D_{2}^{-1}+\frac{P_{3}}{2 \pi i}\left(\int_{C_{6}+C_{3}} \frac{\left[D_{1}\left(g_{c}(\infty)\right)^{-1} D_{2}^{-1} \tilde{g}_{c}(\tau)-I\right] d \tau}{\tau-z}\right)(\zeta) \\
g_{c}(\infty) \doteqdot\left(G_{1} G_{2}\right)^{-1} B^{-1} G_{1}, \quad \tilde{g}_{c}(\zeta) \doteqdot \Omega^{-}(\zeta) D_{2} g_{c}(\zeta) D_{1}^{-1}\left(\Omega^{+}(\zeta)\right)^{-1}
\end{gathered}
$$

In the above $P_{1}, P_{2}, P_{3}$ denote the limit $z \rightarrow \zeta$ from $S_{4}+S_{5}+S_{6}, S_{2}+S_{3}+S_{4}, S_{6}+S_{1}$ $+S_{2}$, respectively; for example $P_{1}\left(\int_{C_{4}+C_{1}} \frac{g(\tau)}{\tau-z} d \tau\right)(\zeta)=\int_{C_{4}+C_{1}} \frac{g(\tau)}{\tau-\zeta+i 0} d \tau$.

Proof. Considering (4.7) and using Plemelj's formula it follows that

$$
f(z)=I+\frac{1}{2 \pi i} \int_{C_{4}+C_{1}} \frac{f_{2}(\tau)\left(g_{a}(\tau)-I\right) d \tau}{\tau-z} .
$$


Equation (4.50) follows by taking the limit of (4.53) as $z$ approaches $C_{4}+C_{1}$ from below. Equations (4.51), (4.52) are obtained in a similar way. Q.E.D.

It should be noted that appropriate use of Plemelj's formulae yields directly integral representations for the function $\Psi(z)$. In particular (4.2)-(4.4) imply

$$
\begin{array}{cl}
\Psi_{2}^{-}(\zeta)=\Psi_{1}^{+}(\zeta)\left(I+\Gamma_{1}(\zeta)\right), \zeta \text { on } C_{2} ; & \Psi_{4}^{-}(\zeta)=\Psi_{5}^{+}(\zeta)\left(I+\Gamma_{4}(\zeta)\right), \zeta \text { on } C_{5} ; \\
\Psi_{2}^{-}(\zeta)=\Psi_{3}^{+}(\zeta)\left(I+\Gamma_{2}(\zeta)\right), \zeta \text { on } C_{3} ; & \Psi_{6}^{-}(\zeta)=\Psi_{5}^{+}(\zeta)\left(I+\Gamma_{5}(\zeta)\right), \zeta \text { on } C_{6} ; \\
\Psi_{4}^{-}(\zeta)=\Psi_{3}^{+}(\zeta)\left(I+\Gamma_{3}(\zeta)\right), \zeta \text { on } C_{4} ; & \Psi_{6}^{-}(\zeta)=\Psi_{1}^{+}(\zeta)\left(I+\Gamma_{6}(\zeta)\right), \zeta \text { on } C_{1}, \\
\Psi(z) \rightarrow I \quad \text { as } z \rightarrow \infty,
\end{array}
$$

where the superscripts are used to remind us that, for the choice of arrows of Fig. 2, $\Psi_{1}, \Psi_{3}, \Psi_{5}$ are (+) functions while $\Psi_{2}, \Psi_{4}, \Psi_{6}$ are (-) functions; also

$$
\begin{gathered}
\left(\Gamma_{1}\right)_{21}=a e^{2 \theta}, \quad\left(\Gamma_{2}\right)_{12}=-b e^{-2 \theta}, \quad\left(\Gamma_{3}\right)_{21}=c e^{2 \theta}, \quad\left(\Gamma_{4}\right)_{12}=-a e^{-2 \theta}, \\
\left(\Gamma_{5}\right)_{21}=b e^{2 \theta}, \quad\left(\Gamma_{6}\right)_{12}=-c e^{-2 \theta},
\end{gathered}
$$

and all the other elements of the matrices $\Gamma_{j}$ equal zero. Equations (4.54) yield

$$
\Psi(z)=I-\frac{1}{2 \pi i} \int_{C} \frac{\Psi^{+}(\tau) \Gamma(\tau) d \tau}{\tau-z},
$$

where $\Gamma(\tau)$ equals $\Gamma_{1}, \Gamma_{2}, \ldots, \Gamma_{6}$ on $C_{2}, C_{3}, \ldots, C_{1}$, respectively. By taking the limits of (4.55) as $z$ approaches $C$ from $S_{1}, S_{3}, S_{5}$ one may obtain a system of integral equations, which of course will be discontinuous at the origin. The results of Theorem 1, and Proposition 1 may be interpreted as providing a constructive approach to mapping the above linear integral equations to regular Fredholm equations. However, the representation (4.55) is still useful for obtaining a formula for $q(t)$ : Taking the large $z$ limit of Eq. (4.55) and using (3.4) it follows that

$$
q(t)=\frac{1}{\pi}\left\{\int_{C} \Psi^{+}(\tau) \Gamma(\tau) d \tau\right\}_{12} .
$$

Let $q_{v}(t)$ denote the solution of P II in the form (1.1). It is well established in the literature [6] that there exists an explicit transformation relating $q_{1-v}(t)$ and $q_{v}(t)$; also $q_{-v}(t)=q_{v}(-t)$. Hence, the solution to the initial value problem of P II with any $v$ can be reduced to one with $0 \leqq v<1$, which then can be solved according to the results of Theorems 1 and 2 . It is interesting that the above relationship between $q_{v}$ and $q_{1-v}$ follows naturally from the RH problem point of view:

\section{B. $1 \leqq v$}

In this case the RH problem defined by (4.2)-(4.4) is singular, since $\Psi(z)$ has a nonintegrable singularity at the origin. Consider for concreteness the case that $1 \leqq v<2$. Then the singular part of $\Psi_{1}$ is

$$
\frac{X_{v}}{z^{v}}, X_{v} \doteqdot \frac{e^{-u(t)}}{\sqrt{2}}\left(\begin{array}{rr}
\alpha & \beta \\
-\alpha & -\beta
\end{array}\right) .
$$


Thus the singular parts of $\Psi_{2}, \ldots, \Psi_{6}$ are $X_{v} G_{1} / z^{v}, \ldots, X_{v} G_{1} G_{2} G_{3} G_{4} G_{5} / z^{v}$, respectively. Let us introduce the sectionally analytic function $\Psi(z)$ via

where $T_{1}(z)$ equals

$$
\tilde{\Psi}(z) \doteqdot \Psi(z)-\frac{X_{v}}{z^{v}} T_{1}(z)
$$

$$
I, G_{1}, G_{1} G_{2}, G_{1} G_{2} G_{3}, G_{1} G_{2} G_{3} G_{4}, G_{1} G_{2} G_{3} G_{4} G_{5},
$$

for $z$ in $S_{1}, S_{2}, \ldots, S_{6}$, respectively. It is clear that $\tilde{\Psi}(z)$ will have at most an integrable singularity at the origin and hence it will satisfy a regular $\mathrm{RH}$ problem. Actually, using (4.58) in (4.54) it follows that $\tilde{\Psi}(z)$ satisfies

$$
\tilde{\Psi}^{-}(\zeta)=\tilde{\Psi}^{+}(\zeta)(I+\Gamma(\zeta))+\Delta(\zeta), \zeta \quad \text { on } \quad C ; \quad \tilde{\Psi}(z) \rightarrow I \quad \text { as } \quad z \rightarrow \infty, \text { (4.59a) }
$$

where $\Delta(\zeta)$ is defined by $\Delta(\zeta)=X_{v} T_{2}(\zeta) / \zeta^{v}$, and $T_{2}(\zeta)$ equals

$$
\begin{gathered}
g_{1}(\zeta)-G_{1}, \quad G_{1} G_{2}\left(g_{2}(\zeta)\right)^{-1}-G_{1}, \quad G_{1} G_{2} g_{3}(\zeta)-G_{1} G_{2} G_{3}, \\
G_{1} G_{2} G_{3} G_{4}\left(g_{4}(\zeta)\right)^{-1}-G_{1} G_{2} G_{3}, \\
G_{1} G_{2} G_{3} G_{4} G_{5}(\zeta)-G_{1} G_{2} G_{3} G_{4} G_{5}, \quad\left(g_{6}(\zeta)\right)^{-1}-e^{-2 i \pi v} G_{1} G_{2} G_{3} G_{4} G_{5},
\end{gathered}
$$

for $\zeta$ on $C_{2}, C_{3}, \ldots, C_{6}, C_{1}$, respectively. Note that $\Delta(\zeta)$ is regular at the origin; this is the case even for $\zeta$ on $C_{1}$ by virtue of the fact that $X_{v}$ is a singular matrix and using (3.9).

Equations (4.59a) define an inhomogeneous, regular RH problem. Its solution can be expressed in terms of the underlying homogeneous problem,

$$
\phi^{-}(\zeta)=\phi^{+}(\zeta)(I+\Gamma(\zeta)), \zeta \quad \text { on } \quad C ; \quad \phi(z) \rightarrow I \quad \text { as } \quad z \rightarrow \infty .
$$

The RH problem (4.60) can be solved via the methods of Theorems 1 and 2. Actually $\phi(\zeta)$ can also be related to a monodromy problem. It is clear that both $\psi_{v-1}(z)$ and $\psi_{1-v}(z)$ satisfy $(4.60)$ and have the required behavior at the origin. However, the direct problem for $\psi_{v}(z)$ implies $a+b+c+a b c=-2 i \sin v \pi$ [see (3.9)], which suggests that $\phi(z)=\psi_{1-v}(z)$. Solving (4.60) for $I+\Gamma(\zeta)$ in terms of $\psi_{1-v}(z)$, substituting this expression in (4.59a), and using Plemelj's formula it follows that

$$
\psi_{v}(z)=\frac{X_{v}}{z^{v}} T_{1}(z)+\psi_{1-v}(z)-\frac{1}{2 \pi i} \int_{C} \frac{\Delta(\tau)\left(\psi_{1-v}(\tau)\right)^{-1}}{\tau-z} \psi_{1-v}(z) .
$$

Equation (4.61) provides the solution of $\psi_{v}(z)$ explicitly in terms of the solution of $\psi_{1-v}(z)$. However, one may avoid the integrals by multiplying (4.61) by the singular matrix with one in each entry:

$$
\left(\begin{array}{ll}
1 & 1 \\
1 & 1
\end{array}\right) \psi_{v}(z)=\left(\begin{array}{ll}
1 & 1 \\
1 & 1
\end{array}\right) \psi_{1-v}(z)
$$

Equation (4.61) is valid even if $v \geqq 2$. In this case both $\Delta(\zeta)$ and $\psi_{1-v}(z)$ are singular. However, the integral appearing in (4.61) is still well defined.

Using Eq. (4.62), the symmetry relationships $\left(\psi_{v}(z)\right)_{12}=\left(\psi_{v}(-z)\right)_{21},\left(\psi_{v}(z)\right)_{22}$ $=\left(\psi_{v}(-z)\right)_{11}$, and Eq. (1.2), one may obtain explicitly $\psi_{v}(z)$ in terms of $\psi_{1-v}(z)$ [37]. Furthermore Eq. (4.62) can be used for deriving the explicit relationship between $q(t)$ and $q_{1-v}(t)$ mentioned above. 
Lemma 5. The large and small $z$ limit of (4.62) yield

and

$$
K_{1-v}(t)-q_{1-v}(t)=K_{v}(t)-q_{v}(t),
$$

$$
e^{u_{1}-v(t)}=\lambda e^{-u_{v}(t)} \Lambda_{v}(t),
$$

respectively, where $K(t), \Lambda(t)$, and $u(t)$ are defined in (3.5), (3.6), and $\lambda$ is a constant parameter. Both of the above equations imply

$$
q_{1-v}(t)=q_{v}(t)+\frac{1-2 v}{2 q_{v}^{2}(t)+2 q_{v t}(t)+t} .
$$

Proof. The first part of the Lemma 5 follows easily from (4.61), (3.5), (3.6). To derive (4.6) from (4.63) note that $K_{t}(t)=-q^{2}(t)$. Hence (4.63) implies $q_{1-v}(t)=q_{v}(t)$ $+q_{v}^{2}(t)-q_{1-v}^{2}(t)$. Using this equation in (4.63) we obtain (4.65). Similarly differentiating (4.64) and using $\Lambda_{t}(t)-2 q(t) \Lambda(t)=1-2 v$, we again obtain (4.65). Q.E.D.

\section{Appendix A}

In this appendix we investigate further the origin condition (4.6). This condition implies Eqs. (3.9) within a choice of signs, actually (4.6) implies

$$
a+b+c+a b c=2 i \sigma \sin v \tau, \sigma= \pm 1 .
$$

It was shown in Theorem 1 that (4.6) follows naturally from the RH problem formulation of $\Psi(z)$. On the other hand this $\mathrm{RH}$ problem is equivalent to a system of linear integral equations [see the discussion after (4.55)]. These equations follow from the integral representation (4.55). In this appendix it is shown that an investigation of Eq. (4.55) in the neighborhood of the origin also yields (A.1). In this respect the following result is useful.

Proposition 2. Consider the Cauchy type integral

$$
\Phi(z) \doteqdot \frac{1}{2 \pi i} \int_{C} \frac{\phi(\tau) d \tau}{\tau^{v}(\tau-z)},
$$

where $\phi(\tau)$ is a Hölder function on $C$ and $\phi(\tau)$ tends to $\phi_{1}, \ldots, \phi_{6}$ as $\tau$ tends to zero along $C_{1}, \ldots, C_{6}$, and $C$ is defined in Fig. 2. Then

where $\alpha_{j}=A_{j} /(2 i \sin v \pi)$ and

$$
\Phi(z) \sim \frac{\alpha_{j}}{z^{v}}, z \rightarrow 0 \quad \text { in } S_{j}, \quad j=1, \ldots, 6,
$$

$$
\begin{aligned}
& A_{1}=v_{1} \phi_{1}+v_{2}\left(-\phi_{2}+\phi_{3}-\phi_{4}+\phi_{5}-\phi_{6}\right), \\
& A_{2}=v_{1}\left(\phi_{1}-\phi_{2}\right)+v_{2}\left(\phi_{3}-\phi_{4}+\phi_{5}-\phi_{6}\right), \\
& A_{3}=v_{1}\left(\phi_{1}-\phi_{2}+\phi_{3}\right)+v_{2}\left(-\phi_{4}+\phi_{5}-\phi_{6}\right), \\
& A_{4}=v_{1}\left(\phi_{1}-\phi_{2}+\phi_{3}-\phi_{4}\right)+v_{2}\left(\phi_{5}-\phi_{6}\right), \\
& A_{5}=v_{1}\left(\phi_{1}-\phi_{2}+\phi_{3}-\phi_{4}+\phi_{5}\right)-v_{2} \phi_{6}, \\
& A_{6}=v_{1}\left(\phi_{1}-\phi_{2}+\phi_{3}-\phi_{4}+\phi_{5}-\phi_{6}\right),
\end{aligned}
$$

where $v_{1} \doteqdot \exp (v \pi i), v_{2} \doteqdot \exp (-v \pi i)$. 
Proof. From the general theory of (A.2) [27] it is known that the behavior of $\Phi(z)$ has the form (A.2) in the neighborhood of the origin. To determine the constants $\alpha_{j}$ use Plemelj's formulae: They yield

$$
\alpha_{1}-\alpha_{2}=\phi_{2}, \alpha_{3}-\alpha_{2}=\phi_{3}, \alpha_{3}-\alpha_{4}=\phi_{4}, \alpha_{5}-\alpha_{4}=\phi_{5}, \alpha_{1}-\alpha_{2} e^{-2 \pi v}=\phi_{1} \text {. }
$$

Equations (A.4) imply (A.3). Q.E.D.

Using the above and Eq. (4.55) it follows that:

Proposition 3. Assume that $\Psi(z)$, which solves Eq. (4.55), has the following dominant behavior at the origin

$$
\Psi(z) \sim\left(\begin{array}{cc}
A & B \\
\tilde{A} & \tilde{B}
\end{array}\right) \frac{1}{z^{v}}, z \rightarrow 0 \quad \text { in } \quad S_{1} ; \quad 0<v<1 .
$$

Then

$$
\begin{gathered}
a+b+c+a b c=2 i \sigma \sin v \pi, \quad \sigma= \pm 1, \\
\frac{A}{B}=\frac{\tilde{A}}{\tilde{B}}=\sigma \frac{1+a b}{e^{i \pi v}-a b}=\frac{1-\sigma a e^{-i \pi v}}{c+\sigma e^{-i \pi v}} .
\end{gathered}
$$

Proof. To find the dominant behavior of (4.55) as $z \rightarrow 0$, say in $S_{1}$, one needs to compute $\Psi^{+}$and $F$ in the neighborhood of the origin; this is straightforward. Then using Proposition 2 it follows that

$$
\begin{aligned}
\left(\begin{array}{cc}
A & B \\
\tilde{A} & \tilde{B}
\end{array}\right)= & \frac{1}{2 i \sin v \pi}\left\{v_{1} c\left(\begin{array}{cc}
0 & A \\
0 & \tilde{A}
\end{array}\right)+v_{2} a\left(\begin{array}{cc}
B & 0 \\
\tilde{B} & 0
\end{array}\right)+v_{2} b\left(\begin{array}{cc}
0 & A+a B \\
0 & \tilde{A}+a \tilde{B}
\end{array}\right)\right. \\
& +v_{2} c\left(\begin{array}{cc}
A B+B(1+a b) & 0 \\
\tilde{A} B+\tilde{B}(1+a b) & 0
\end{array}\right)+v_{2} a\left(\begin{array}{cc}
0 & A(1+b c)+B(\varrho-b) \\
0 & \tilde{A}(1+b c)+\tilde{B}(\varrho-b)
\end{array}\right) \\
& \left.+v_{2} b\left(\begin{array}{cc}
A(\varrho-c)+B(1+a \varrho) & 0 \\
\tilde{A}(\varrho-c)+\tilde{B}(1+a \varrho) & 0
\end{array}\right)\right\},
\end{aligned}
$$

where

$$
\varrho \doteqdot a+b+c+a b c .
$$

Equation (A.8) implies (A.6), (A.7). Q.E.D.

The direct problem implies that the singular part of $\Psi(z)$ is given by (4.57) where $\alpha, \beta$ are related to $a, b, c$ through Eqs. (3.9). Manipulating these equations it follows that $\alpha / \beta$ is independent of $\hat{\Lambda}$; actually

$$
\frac{\alpha}{\beta}=\frac{1+a b}{a b-e^{i \pi v}},
$$

which agrees with (A.7) within the sign of $\sigma$.

\section{Appendix B}

In this appendix we compare the approach used to solve the inverse problem here, with that used in [20]. For concreteness we concentrate on the case that $v$ is an integer 


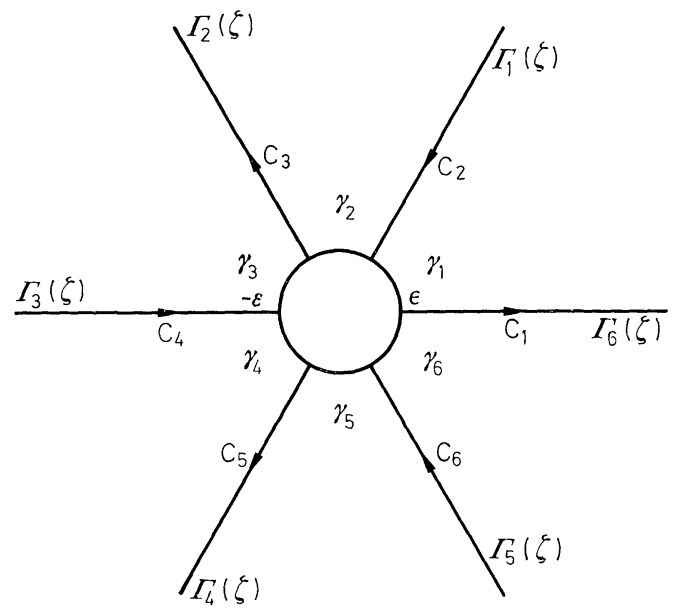

Fig. 6

The jump conditions (4.54) imply

$$
\Psi^{+}(\zeta)=\Psi^{-}(\zeta)(I-\Gamma(\zeta)), \zeta \quad \text { on } \quad C, \quad \Psi(z) \rightarrow I \quad \text { as } \quad z \rightarrow \infty,
$$

where $\Gamma(\zeta)$ is defined by (4.55). If $v>0$ the $\mathrm{RH}$ problem (B.1) is singular, hence to solve it, one needs first to subtract its singular part. However, one can still give an integral representation for $\Psi(z)$, analogous to the formula (4.55). Actually, using Plemelj's formulae, (B.1) yields

$$
\Psi(z)=I-\frac{1}{2 \pi i} \int_{C} \frac{\Psi^{-}(\tau) \Gamma(\tau) d \tau}{\tau-z}-\frac{1}{2 \pi i} \int_{\gamma_{1}+\ldots+\gamma_{6}} \frac{\Psi(\tau) d \tau}{\tau-z} .
$$

Equation (B.2) should be interpreted in the Hadamand sense: Both integrals appearing in (B.2) in general diverge, but their sum converges. For example if $v=1$, the second integral of (B.2) converges; the first integral of (B.2) also converges because the coefficient of $\ln \varepsilon$ appearing in this integral is zero in light of (3.9). For $v=2$ both integrals diverge but their sum converges. (Details can be found in [37].)

Writing the first vector component of (B.2) we obtain

$$
\begin{aligned}
\underline{\Psi}^{(1)}(z)= & \left(\begin{array}{l}
1 \\
0
\end{array}\right)-\frac{1}{2 \pi i}\left(a \int_{C_{2}} \underline{\Psi}_{2}^{(2)}(\tau)+c \int_{C_{4}} \underline{\Psi}_{4}^{(2)}(\tau)+b \int_{C_{6}} \underline{\Psi}_{6}^{(2)}(\tau)\right) \frac{e^{2 \theta(\tau)} d \tau}{\tau-z} \\
& -\frac{1}{2 \pi i} \int_{\gamma_{1}+\ldots+\gamma_{6}} \frac{\underline{\Psi}^{(1)}(\tau) d \tau}{\tau-z} .
\end{aligned}
$$

Equation (B.3) is valid (in the Hadamand sense) for all $z$. If one restricts $z$ in $S_{1}$, one obtains the equation obtained by adding (3.32a)-(3.32f) of [20]. Using (4.54) and (A.1) in this equation one obtains the main equation of the inverse problem of [20], namely

$$
\begin{aligned}
\underline{\Psi}_{1}^{(1)}(z)= & \left(\begin{array}{l}
1 \\
0
\end{array}\right)-\frac{1}{2 \pi i}\left(a \int_{C_{42}} \underline{\Psi}_{1}^{(2)}(\tau)+b \int_{C_{46}} \underline{\Psi}_{1}^{(2)}(\tau)-c b \int_{C_{46}} \underline{\Psi}_{1}^{(1)}(\tau)\right) \frac{e^{2 \theta(\tau)} d \tau}{\tau-z} \\
& -\frac{1}{2 \pi i} \int_{\gamma_{1}+\ldots+\gamma_{6}} \frac{\underline{\Psi}_{1}^{(1)}(\tau) d \tau}{\tau-z}
\end{aligned}
$$


where the contours $C_{42}, C_{46}$ are given in Fig. 7. In a

Fig. 7

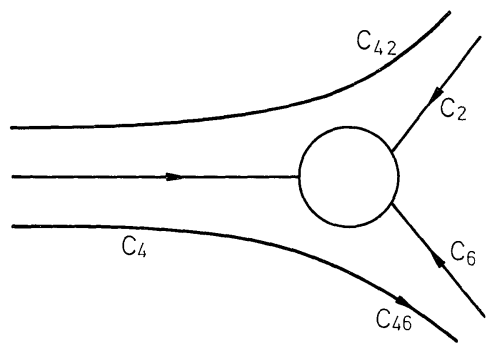

similar way one obtains an integral representation for $\Psi_{1}^{(2)}(z)$.

Equation (B.4) is not singular. However, in order to obtain an integral equation from (B.4) one must "project," i.e. take the limit of $z$ as $z$ approaches the contours $C_{42}$ and $C_{46}$. But (B.4) is valid only for $z$ in $S_{1}$. Thus it seems to us that one has to extract some more information from (B.3). The techniques developed in this paper are dealing exactly with this problem: Through induction and a series of transformations one can map the integral representation (B.3) to the three Fredholm integral Eqs. (4.50)-(4.52).

Acknowledgements. This work was partially supported by the Office of Naval Research under Grant Number N00014-76-C-0867, the National Science Foundation under Grant Number MCS-8202117, and the Air Force Office of Scientific Research under Grant Number 78-3674-D.

\section{References}

1. Painlevé, P.: Bull. Soc. Math. Fr. 28, 214 (1900) Painlevé, P.: Acta Math. 25, 1 (1902)

2. Gambier, B.: Acta Math. 33, 1 (1909)

3. Ince, E.L. : Ordinary differential equations (1927). New York: Dover 1956

4. The work done in Soviet Union up to 1976 is reviewed by the editor of Diff. Urav. in the following two review articles (where he quotes 127 references):

1) Erugin, N.P.: Diff. Urav. 12, 387 (1976)

2) Erugin, N.P.: Diff. Urav. 12, 579 (1976)

5. Gromak, V.I.: Diff. Urav. 14, 2131 (1978)

6. Lukashevich, N.A.: Diff. Urav. 7, 1124 (1971)

7. Gromak, V.I.: Diff. Urav. 11, 373 (1975)

8. Lukashevich, N.A.: Author's abstract of doctoral dissertation (in Russian) (Kiev, 1971)

9. Gromak, V.I.: Diff. Urav. 12, 740 (1976)

10. Fokas, A.S., Yortsos, Y.C.: The transformation properties of the sixth Painlevé equation and oneparameter families of solutions. Lett. Nuovo Cimento 30, 539-540 (1980)

11. Barouch, E., McCoy, B.M., Wu, T.T.: Zero-field susceptibility of the two-dimensional Ising model near $T_{c}$. Phys. Rev. Lett. 31, 1409 (1973)

Wu, T.T., McCoy, B.M., Tracy, C.A., Barouch, E.: Spin-spin correlation functions for the twodimensional Ising model-exact theory in the scaling region. Phys. Rev. B 13, 316 (1976)

12. Jimbo, M., Miwa, T., Mori, Y., Sato, M.: Physica 1D, 80 (1980)

Jimbo, M., Miwa, T. : Proc. Jpn. Acad. 56 A, 405 (1980)

13. Miles, J.W.: Proc. R. Soc. London A 361, 277 (1978)

14. McCoy, B.M., Perk, J.H.H., Schrock, R.: Nucl. Phys. B (1983) and Correlation functions of the transverse Ising chain at the critical field for large temporal and spatial separations 
15. 1) Ablowitz, M.J., Segur, H.: Exact linearization of a Painlevé transcendent. Phys. Rev. Lett. 38, 1103 (1977)

2) Ablowitz, M.J., Ramani, A., Segur, H. : Nonlinear evolution equations and ordinary differential equations of Painlevé type. Lett. Nuovo Cimento 23, 333 (1978)

3) Ablowitz, M.J., Ramani, A., Segur, H.: A connection between nonlinear evolution equations and ordinary differential equations of $P$-type. I. J. Math. Phys. 21, 715 (1980)

4) Ablowitz, M.J., Ramani, A., Segur, H.: A connection between nonlinear evolution equations and ordinary differential equations of $P$-type. II. J. Math. Phys. 21, 1006 (1980)

16. Ablowitz, M.J., Segur, H.: Solitons and the inverse scattering transform. SIAM Stud. Appl. Math. (Studies 4) 1981

17. Fokas, A.S., Ablowitz, M.J.: On a unified approach to transformations and elementary solutions of Painlevé equations. J. Math. Phys. 23, 2033 (1982)

18. Sato, M., Miwa, T., Jimbo, M.: Publ. RIMS, Kyoto Univ. 15, 201 (1979); Proc. Jpn. Acad. 55 A, 267 (1979); In : Lecture Notes in Physics, Vol. 126. Berlin, Heidelberg, New York: Springer 1980, p. 429

19. Ueno, K.: Master's thesis, Kyoto Univ. (1979). Proc. Jpn. Acad. 56 A, 97 (1980); 56 A, 103 (1980)

20. Flaschka, H., Newell, A.C.: Monodromy and spectrum preserving deformations. I. Commun. Math. Phys. 76, 67 (1980)

21. Jimbo, M., Miwa, T., Ueno, K.: Physica 2D, 306 (1981) Jimbo, M., Miwa, T.: Physica 2 D, 407 (1981); 4 D, 47 (1981)

22. Fokas, A.S., Ablowitz, M.J.: Linearization of the Korteweg-deVries and Painlevé. II. Equations. Phys. Rev. Lett. 47, 1096 (1981)

23. Whitham, G.B.: Private communication

24. Fokas, A.S., Ablowitz, M.J.: On the inverse scattering transform for the Kadomtsev-Petviashvili equation. Phys. Lett. 94 A, 67 (1983)

25. Ablowitz, M.J., Bar Yaacov, D., Fokas, A.S.: On the inverse scattering transform for the Kadomtsev-Petviashvili equation. Stud. Appl. Math. (to appear)

26. Fokas, A.S.: On the inverse scattering of first order systems in the plane related to nonlinear multidimensional equations. Phys. Rev. Lett. 51, 3-6 (1983)

Fokas, A.S., Ablowitz, M.J.: On the inverse scattering transform of multi-dimensional nonlinear equations related to first order systems in the plane. INS No. 24, January 1983

27. i) Gakhov, F.D.: Boundary value problems. New York: Pergamon Press 1966

ii) Muskhelishvili, N.I.: Singular integral equations. Groningen : Noordhoff 1953

iii) Vekua, N.P. : Systems of singular integral equations. New York: Gordon and Breach 1967

28. Krein, M.G.: Usp. Mat. Nauk. 13, 3 (1958)

Gohberg, I., Krein, M.G.: Usp. Mat. Nauk. 13, 2 (1958)

Prossdorf, S.: Some classes of singular equations. Amsterdam: North-Holland 1978

29. Riemann, B.: Gesammelte Mathematische Werke. Leipzig (1892)

30. Hilbert, D.: Grundzüge der Integralgleichungen. Leipzig (1924)

31. Birkhoff, G.D.: Trans. AMS 436 (1909) and 199 (1910)

32. Plemelj, J.: Problems in the sense of Riemann and Klein. New York: Wiley 1964

33. Erugin, N.P.: Linear systems of ODE's. New York: Academic Press 1966

34. Chudnovsky, D.V.: In: Bifurcation phenomena in mathematical physics and related topics, p. 385. Bardos, C., Bessis, D. (eds.). Dordrecht, Boston: Reidel 1980

Chudnovsky, D.V., Chudnovsky, G.V.: Lett. Math. Phys. 4, 373 (1980)

35. Miwa, T.: Proc. Jpn. Acad. 56 A, 301 (1980)

36. Beals, R., Coifman, R.R.: Scattering and inverse scattering for first order systems. Preprint, Yale 1982

37. Sanborn, F.: Ph. D. thesis, Clarkson College (1984)

Communicated by A. Jaffe

Received June 15, 1983 
